\title{
General factors that influence the immune responses in children
}

\author{
EUN HEE HA
}

Dulwich College Suzhou

\begin{abstract}
The research paper is written with the aim to identify different factors that influence children's immune responses. Five theories are explored on children's susceptibility to certain diseases and their lack of susceptibility to others. Aspects that are explored in this paper are children's levels of immunoglobulin production, their cytokine production amount, their underdevelopment of receptors, their asymptomatic nature to certain diseases, and environmental factors. Through exploring different studies on child immunity, the paper concludes that the susceptibility of children to diseases largely depends on the type of disease and the individual's immune system. Future research is clearly required to identify a smaller scope of the factors that influence children's immune responses to provide a more detailed reasoning in the causes of children's proneness to certain diseases and their lack of susceptibility in others.
\end{abstract}

\section{Introduction}

\subsection{General}

Children are often prone to gain certain viral infections. They are regarded to be more susceptible to environmental risks and infections. At the same time, children also seem to be less likely to gain certain diseases, presumably due to certain health reasons or other environmental factors. Although these common characteristics of children's immune responses are often identified and noticed, the underlying reasons for these responses remain unclear as children's immune responses to certain infections would largely differ based on the type of infection and the children's own immune system. This article aims to identify the general factors that influence children's proneness to certain types of diseases and their lack of susceptibility to others. Through identifying the general factors that influence children's immune responses, better understanding can be developed on children's immune responses to certain diseases, enabling the causes of these immune responses to be identified and treated.

\subsection{Abbreviations}

Abbreviations used in is paper is listed in alphabetical order below.

ACE2: angiotensin converting enzyme II

COVID-19: 2019 novel coronavirus disease

iL: Interleukin

iL-2: Interleukin-2

iL-4: Interleukin-4

iL-6: Interleukin-6

Ig: Immunoglobulin

IgA: Immunoglobulin A

IgG: Immunoglobulin G

\subsection{Background}

The immune system in the body exists to identify foreign antigens from the body's existing tissues or other components. The body's immunity can be largely divided into three parts: innate immunity, adaptive immunity and passive immunity. Innate immunity is the immunity that humans have when the are born. It creates immune responses that are general and non-specific, targeting all foreign pathogens that enters the body. Examples of innate immunity include physical barriers 
such as the skin and the mucus in cells. Adaptive immunity, also known as acquired immunity, are immune responses developed after an individual is exposed to a disease or infection. Adaptive immunity produces immune responses that are specific and only targets on specific pathogen with foreign antigens. Memory cells developed during an infection will aid the body's immune system to identify the particular foreign pathogen that caused the infection or disease, enabling the body to protect its host and kill the pathogens at a faster rate. Passive immunity is immunity borrowed from another source. An example of this is when infants receive antibodies from the mother's break milk for protection against infections.

\subsection{Immunoglobulins}

Immunoglobulins, also known as antibodies, enable the body to identify foreign pathogens. Generally, immunoglobulins would bind to specific antigenic determinant and effector functions such as fixation of complement or binding to various cell types in the immune system would be carried out. ${ }^{1}$ The immunoglobulins can be classified into five different classes: $\operatorname{IgG}, \operatorname{IgM}, \operatorname{Ig} A, \operatorname{IgD}$ and $\operatorname{IgE}$.

\subsection{Cytokines}

Cytokines are proteins that regulate various inflammatory responses in the body. Types of cytokines include lymphokines, monokines, chemokines and interleukins. ${ }^{2}$ When cytokines are released in the body, they would attach to receptors of the target cells, triggering a chemical signal to be sent to receptor cells which enable white blood cells to protect the host and kill the pathogens that are identified. ${ }^{3}$

\section{Method}

\subsection{General}

In this research paper, theories from other studies will be discussed and evaluated. As no direct experiment is conducted, the paper can only conclude that there are correlations between child immunity and the factors explored in this paper. Future research is required to determine the causal relationships present in child immunity and the different aspects studied.

\footnotetext{
${ }^{1}$ IMMUNOGLOBULINS - STRUCTURE AND FUNCTION, www.microbiologybook.org/mayer/IgStruct2000.htm.

${ }^{2}$ Eustice, Carol. "How Cytokines Play a Significant Role in Inflammatory Response." Verywell Health, Verywell Health, 12 Oct. 2019, www.verywellhealth.com/what-are-cytokines189894.
}

\subsection{Theory}

Several theories are proposed for the various susceptibility children has to different diseases. Reasons for proneness in infections include the fact that $\mathrm{IgG}$ and $\operatorname{IgA}$ production are not fully developed in children. Since immunoglobins are vital for the body to effectively protect the host and kill the pathogens, the low concentration of certain immunoglobulins may be a detrimental factor that impact the immune response of children and their susceptibility to diseases. Another argument for the proneness of children to gain certain infections is that children produce lower levels of cytokines, which are significant for the survival of children during infection. As proteins that regulate the immune system, cytokines are chemicals that would significantly influence an individual's immune response. As such, the low concentration of cytokines in children may also be viewed as a reasonable factor that influence the proneness of children to certain infections.

Several arguments can also be made for children's lack of susceptibility to certain infections. To begin with, children may have underdeveloped receptors that prevent them from gaining the disease. Another argument made is that children might also be as susceptible to certain diseases as adults but would not show any symptoms. As asymptomatic carriers, children would present little to no symptoms, resulting in most cases to be unidentified and noted. Environmental factors may also be a detrimental factor of children's immune responses to certain diseases. Since children are typically less exposed to chemicals or toxic smoke, they will have a healthier body and organ system, causing them to be less susceptible to illnesses.

All five theories seem relatively reasonable with some supporting scientific evidence. Evidence on these theories will be explored and critically evaluated in the next section.

\section{Results and discussions}

\subsection{Low Concentration of Immunoglobulins}

Research presents that children in general produce lower concentrations of $\operatorname{IgG}$ and $\operatorname{IgA}$ in their body, presumably due to the lack of development of their immune system. ${ }^{4}$ When 270 children and 30 adults were studied, quantitative determination of the immunoglobulins showed that IgG and IgA concentrations gradually rose

\footnotetext{
3 "Everything About Cytokines: Their Function, Structure, and Properties." Bodytomy, 21 Mar. 2015, bodytomy.com/cytokines-function-structure-properties.

${ }^{4}$ Stoop, J W, et al. Serum Immunoglobulin Levels in Healthy Children and Adults. 27 May 1968.
} 
with increasing age. IgM concentration also remained constant at distinctly lower levels than in adults. Clearly, there is a correlation between the concentration of immunoglobulins and the age of the participants in the research. Since the number of immunoglobulins would largely influence one's immune response to a specific type of disease, the low concentration of immunoglobulins may be a factor that influence the immune response of children and their proneness to certain diseases.

\subsection{Low Levels of Cytokines}

Research indicates that children in general produce low levels of cytokines. ${ }^{5}$ When ten healthy children (six boys and four girls) and ten adults were studied, the research noted that healthy children differ significantly compared with adults in their ability to produce cytokines, specifically iL- 2 , interferon- $\gamma$, iL-4, and iL- 6 . When stimulated with antigens, the median levels of iL-2 and iL-4 in children remained lower than adult's values whilst iL-6 and interferon- $\gamma$ increased but not significantly.

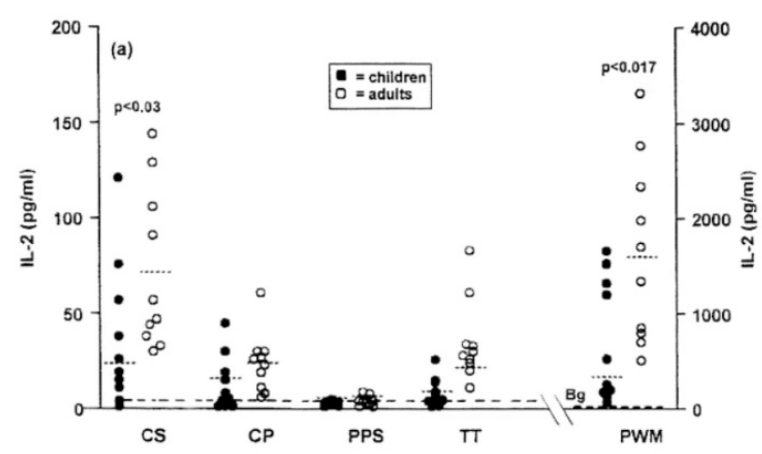

Figure 1. An image presented in the research that discusses the difference in cytokine production between children and adults. The production of IL-2 in adults and children are recorded after stimulation with various antigens and mitogens. ${ }^{6}$

Figure 1 effectively displays that children produce an overall lower concentration of IL-2 in comparison to adults since data points recorded for children's IL-2 levels are generally concentrated at the bottom for all types of antigens and mitogens tested in the study. In contrast, adults have a higher mean IL-2 levels produced with a larger variability for all types of antigens and

\footnotetext{
${ }^{5}$ Lilic, Desa, et al. "Cytokine Production Differs in Children and Adults." Nature News, Nature Publishing Group, www.nature.com/articles/pr19972299.

${ }^{6}$ Lilic, Desa, et al. "Cytokine Production Differs in Children and Adults." Nature News, Nature Publishing Group, www.nature.com/articles/pr19972299.

${ }^{7}$ Shen, Kunling, and Yonghong Yang. Diagnosis, Treatment, and Prevention of 2019 Novel Coronavirus Infection in
}

mitogens tested. Thereby, we can infer from the graph that regardless of the type of antigen or mitogen used, in average adults would have a higher mean production of IL-2 concentration levels than children.

From the study, it is rather apparent that children in the research produced lover levels of cytokines in general regardless of gender. The limitation of the study is that only ten healthy children and ten adults were studied. Since the sample size is relatively small in number, more studies and supporting evidence would be required before low levels of cytokine production can be generalized and applied to all cases of children's immune responses.

\subsection{Underdeveloped receptors}

Children may have receptors that are not fully developed, which impact their immune responses to certain diseases. Take the COVID-19 as an example. Among the 9692 confirmed cases of COVID-19, 28 children aged from 1 month to 17 years old have been reported in China. ${ }^{7}$ Statistically, there seems to be fewer cases of children getting the corona virus and one reason for this is the underdevelopment of ACE2 receptors in children. As ACE2 receptors are not expressed as prominently in children as they are in adults, it would have been harder for the spikes of the viral particles to bind and gain entry to the host cells for replication. ${ }^{8}$ Although the ACE2 receptor is identified to be the main cause for transporting and aiding the corona virus to duplicate, the relationship of ACE2 receptors and children's proneness to the disease was not studied with detail. Since correlation may not always lead to causation, more research should be conducted on the relationship for confirming the theory that underdeveloped receptors may be the cause to children's less susceptibility in certain illnesses.

\subsection{Asymptomatic Carriers}

Individuals who gained the disease but do not show the typical symptoms of the disease are called asymptomatic carriers. Children may simply be asymptomatic carriers: they would be as susceptible to the illnesses as adults, but they may be perceived to be more 'immune' to the illnesses due to the lack of symptoms they show. This might be the case for some children during the COVID-

Children: Experts' Consensus Statement. 7 Feb. 2020, Diagnosis, treatment, and prevention of 2019 novel coronavirus infection in children: experts' consensus statement. 8 Nyt-Syndication. "Children and Coronavirus: Research Finds Some Become Seriously Ill." Boston.com, The Boston $\begin{array}{lll}\text { Globe, } 18 & \text { Mar. }\end{array}$ www.boston.com/news/health/2020/03/18/childrencoronavirus. 
19 outbreak. Research indicated that children are as susceptible to the COVID-19 as adults. ${ }^{9}$ Nevertheless, the cases of children getting COVID-19 is significantly lower than cases of adults gaining the COVID-19, presumably because children who gained he COVID-19 would typically show little to no symptoms. There is actually a case where a 10 -year-old boy is diagnosed to have COVID-19 even though he showed no symptoms to the disease. ${ }^{10}$ Though this may be true, since asymptomatic carriers are not often identified and diagnosed, there is not precise recorded number of asymptomatic carriers in children. As such, no conclusion can be drawn on the asymptomatic nature of children to COVID-19 as there is no certainty on whether the children are actually less susceptible to the disease or are just asymptomatic.

\subsection{Environmental Factors}

Children are often less exposed to toxic chemicals or smoke. As this is the case, they often have healthier organs and tissues which lower their susceptibility to certain diseases. Asthma, for instance, is triggered by air pollution, mold, allergens, tobacco smoke and other exposures. Infants living in homes with high levels of mold were researched to be more likely to develop asthma by the age of $7.1^{11}$ There is clearly a correlation between children's health and the environmental factors they are exposed to. As such, environmental factors could be regarded as one of the factors that influence children's immune responses.

\section{Conclusion}

Overall, the susceptibility of children to diseases largely depends on the types of disease and the individual's immune system. However, generally children's underdevelopment in their immune system may make them more prone to certain diseases. Children's lack of susceptibility to diseases may be cause by their asymptomatic nature to the disease. They may also have underdeveloped receptors that prevent the further spread and replication of the pathogens in their body. Depending on the environment children are exposed to, they may be more prone to certain illnesses and less susceptible to others.

Clearly, not enough research is conducted on the factors that cause children's various immune responses

\footnotetext{
${ }^{9} \mathrm{Bi}$, Qifang, et al. "Epidemiology and Transmission of COVID-19 in Shenzhen China: Analysis of 391 Cases and 1,286 of Their Close Contacts.” MedRxiv, Cold Spring Harbor Laboratory Press, 1 Jan. 2020, www.medrxiv.org/content/10.1101/2020.03.03.20028423v2.

10 Gale, Jason, and Bloomberg News. "Asymptomatic Infection: Doctors Treat 10-Year-Old Boy 'Shedding Virus
}

to different diseases and infections as no definite conclusions can be drawn from existing data and research. This research paper only broadly pointed out the factors that may influence children's immune responses to various diseases. As such, further research should be conducted to identify a smaller scope of the factors that influence children's immune responses to provide a more detailed reasoning in the causes of children's proneness to certain diseases and their lack of susceptibility in others. By identifying specific and more detailed reasonings to the factors that cause children's immune responses, scientists would be able to develop a better understanding of the human immune system in general. Faster and more effective treatments could be provided to young patients to aid their recovery in health and wellbeing.

\section{Acknowledgement}

This research paper would not have been possible without the support of Dr. John D. Lokie. His course on immunology enabled me to grasp some understanding on the basic immune responses individuals may have when encountering an infection or illness. His enthusiasm and knowledge eventually provided me with the inspiration and motivation to write this paper.

I would also like to express my appreciation to Sarah, who was the teacher assistant during the immunology course taught by professor Lokie. Her advice provided me with insights to edit and finish this paper.

\section{References}

1. IMMUNOGLOBULINS - STRUCTURE AND FUNCTION, www.microbiologybook.org/mayer/IgStruct2000.h tm.

2. Eustice, Carol. "How Cytokines Play a Significant Role in Inflammatory Response." Verywell Health, Verywell Health, 12 Oct. 2019, www.verywellhealth.com/what-are-cytokines189894

3. "Everything About Cytokines: Their Function, Structure, and Properties." Bodytomy, 21 Mar. 2015, bodytomy.com/cytokines-function-structureproperties.

without Symptoms'." National Post, 29 Jan. 2020, nationalpost.com/news/world/doctors-say-10-year-old-boycarried-coronavirus-but-exhibited-no-symptoms.

${ }^{11}$ Children's Health: Why the Environment Matters. National Institute of Environmental Health Sciences, www.niehs.nih.gov/health/materials/fact_sheet_childrens_he alth_why_the_environment_matters_508.pdf. 
4. Stoop, J W, et al. Serum Immunoglobulin Levels in Healthy Children and Adults. 27 May 1968.

5. Lilic, Desa, et al. "Cytokine Production Differs in Children and Adults." Nature News, Nature Publishing Group, www.nature.com/articles/pr19972299.

6. Shen, Kunling, and Yonghong Yang. Diagnosis, Treatment, and Prevention of 2019 Novel Coronavirus Infection in Children: Experts' Consensus Statement. 7 Feb. 2020, Diagnosis, treatment, and prevention of 2019 novel coronavirus infection in children: experts' consensus statement.

7. Nyt-Syndication. "Children and Coronavirus: Research Finds Some Become Seriously Ill." Boston.com, The Boston Globe, 18 Mar. 2020, www.boston.com/news/health/2020/03/18/children -coronavirus.
8. Bi, Qifang, et al. "Epidemiology and Transmission of COVID-19 in Shenzhen China: Analysis of 391 Cases and 1,286 of Their Close Contacts." MedRxiv, Cold Spring Harbor Laboratory Press, 1 Jan. 2020, www.medrxiv.org/content/10.1101/2020.03.03.20 $028423 \mathrm{v} 2$.

9. Gale, Jason, and Bloomberg News. "Asymptomatic Infection: Doctors Treat 10-Year-Old Boy 'Shedding Virus without Symptoms'." National Post, 29 Jan. 2020, nationalpost.com/news/world/doctors-say-10-yearold-boy-carried-coronavirus-but-exhibited-nosymptoms.

10. Children's Health: Why the Environment Matters. National Institute of Environmental Health Sciences,

www.niehs.nih.gov/health/materials/fact sheet chi ldrens_health_why_the_environment_matters_ 508 .pdf. 